\title{
Investigation of the effects of temperature and time on reduction of graphene oxide by microwave hydrothermal reactor
}

\author{
SALIMEH KIMIAGAR ${ }^{1, *}$, NASIM RASHIDI ${ }^{1}$ and EHSAN EZZATPOUR GHADIM ${ }^{\mathbf{2}}$ \\ ${ }^{1}$ Department of Physics, Islamic Azad University, Central Tehran Branch (IAUCTB), Tehran 14676-6831, Iran \\ ${ }^{2}$ Young Researchers and Elite Club, Central Tehran Branch, Islamic Azad University, Tehran 13185-768, Iran
}

MS received 16 January 2015; accepted 6 July 2015

\begin{abstract}
Graphene oxide (GO) sheets were synthesized by modified Hummers method. Microwave hydrothermal reactor (MHR) was applied to reduce graphene oxide. Different temperatures 50 and $180^{\circ} \mathrm{C}$ were applied to fabricate four samples with 4, 10, 25 and 60 min exposure times. The deoxygenation of the GO sheets after exposure to MHR was revealed by using UV-visible, Fourier transform infrared, X-ray photoelectron spectroscopy (XPS), thermogravimetric analysis (TGA) and Raman spectroscopy. Based on XPS analysis, the O/C ratio of the GO sheets decreased from 49 to $17 \%$ after exposure to MHR. On the other hand, characterization by TGA illustrated that the O/C ratio of the GO dramatically decreases after exposure of MHR and reached from 37 to $7 \%$. Raman spectroscopy demonstrated that there was no increase in defects' density after reduction. The results confirmed that sample with $180^{\circ} \mathrm{C}$ exposure temperature and $60 \mathrm{~min}$ exposure time was reduced completely. Therefore this can help to advance using MHR as the green technique of GO reduction.
\end{abstract}

Keywords. Graphene; nanostructures; microwave hydrothermal reactor; reduction.

\section{Introduction}

As a rising star in carbon family, graphene has attracted a great attention in recent years due to its excellent conductivity, superior chemical stability and high specific surface area. Graphene oxide (GO) applications have improved in many different kinds of categories such as biochemistry, ${ }^{1}$ drug delivery systems, ${ }^{2}$ electronic, ${ }^{3}$ sensors,${ }^{4}$ mechanic and membranes. ${ }^{5}$

Graphene sheets have been prepared by a variety of techniques such as physical and chemical methods. ${ }^{6,7}$ Both methods have positive and negative points in the synthesis of the $\mathrm{GO}$ with high purities and large-scale production. ${ }^{8}$ Because of the attached oxygen functional groups, GO is electrically insulating and several methods for reduction of GO have been developed to repair change to improve its electrical conductivity. Hence, the chemical and thermal methods are suggested for reduction procedure in order to obtain pure graphene. ${ }^{9}$ Chemical reduction using agents such as hydrazine or dimethyl hydrazine, ${ }^{10,11}$ hydroquinone ${ }^{12}$ and $\mathrm{NaBH}_{4}{ }^{13}$ have been used to reduce GO (RGO). Electrochemical reduction is also carried out for GO reduction. ${ }^{14}$ On the other hand, the strong chemical reducer, e.g., hydrazine, is known as hazardous agent for human's life and environment. In addition, reduction by hydrazine resulted in the formation of $\mathrm{sp}^{3} \mathrm{C}-\mathrm{N}$ bonds as modification disorders on the surface of the reduced sheets. Such disorders prevent obtaining graphene sheets with high electrical conductivities. ${ }^{15}$ So

*Author for correspondence (kimia@khayam.ut.ac.ir) far, some environment-friendly methods for the reduction of GO such as flash photoreduction, ${ }^{16}$ laser irradiation, ${ }^{17} \mathrm{UV}$ irradiation, ${ }^{18}$ commercial microwave oven ${ }^{19}$ and hydrothermal dehydration ${ }^{20}$ have been suggested as suitable substitutions for the common reduction methods.

The hydrothermal technique is becoming one of the most important tools for advanced materials for a wide variety of technological applications such as synthesis of nanoparticle and reduced graphene. The hydrothermal technique exhibits a number of favourable attributes, to mention low energy consumption and low environment pollution. Microwave hydrothermal reactor applies to carry out nanoparticles' synthesis since some of the product properties, like grain shapes, crystallite size distribution and nucleation are precisely controlled. Many reports have shown its considerable advantages when synthesis of nanoparticles is concerned..$^{21}$ It allows a preservation of high purity products, since the reaction mixture do not have contact with the heating elements of reactor. In the traditional 'microwave oven' the microwaves are heating directly the material. In case of the hydrothermal reactor (ERTEC Magnum II) the microwaves are heating both the solvent (water) and material. In some cases, microwave reactor has been equipped by gas fluency such as nitrogen and argon in order to assist better reduction..$^{22,23}$ RGO has also been prepared by directly heating natural graphite with nitric acid and potassium permanganate in a microwave oven. ${ }^{24}$

In this study the facile and straightforward method describes the reduction of GO sheets. Microwave hydrothermal reactor (MHR) with different conditions such as temperature and time is applied. Based on this method the GO 
samples are put into the reactor without using chemical surfactants. In addition, the reduction process does not need gas fluency, hence this method can be efficient and inexpensive. This method can reduce GO sheets in a short time and moderate temperature with no toxic and harmful gas and safe for environment.

\section{Experimental}

Graphite flakes (Sigma-Aldrich, cat \#332461, $150 \mu \mathrm{m}$ lateral dimensions) were used as the raw materials for the synthesis of graphite oxide using a modified Hummers method. ${ }^{8}$

Three grams of graphite was dissolved in a solution containing $180 \mathrm{ml}$ of $\mathrm{H}_{2} \mathrm{SO}_{4}$ and $20 \mathrm{ml}$ of $\mathrm{H}_{3} \mathrm{PO}_{4}$. Then, $\mathrm{KMnO}_{4}$ $(9 \mathrm{~g})$ was slowly added into the mixture. Although temperature of the exothermic reaction was $40-45^{\circ} \mathrm{C}$, the mixture was heated to $55^{\circ} \mathrm{C}$ and stirred for $10 \mathrm{~h}$. Then, the mixture was cooled to room temperature in an ice bath. The reaction was completed by adding $6 \mathrm{ml}$ of $\mathrm{H}_{2} \mathrm{O}_{2}$ (30\%) into the mixture. The solution was centrifuged at $6000 \mathrm{rpm}$ for $6 \mathrm{~h}$ to remove the supernatants. The remaining solid material was washed with $200 \mathrm{ml}$ of $\mathrm{HCl} 30 \%, 200 \mathrm{ml}$ of ethanol $70 \%$ aqueous solution and $200 \mathrm{ml}$ of distillate water (with resistivity $>18 \mathrm{M} \Omega \mathrm{cm})(2 \times)$. The obtained residual material was dispersed in distillate water with ultra-sonication for $5 \mathrm{~h}$, and centrifuged again at $6000 \mathrm{rpm}$ for $5 \mathrm{~h}$ to remove the supernatants. The remaining material was coagulated with $100 \mathrm{ml}$ di-ethyl ether, and the resulting suspension was filtered by a polytetrafluoroethylene membrane (PTFE- 0.22 $\mu \mathrm{m}$ pore size). The filtered powder was dried in vacuum-oven at $65^{\circ} \mathrm{C}$ and $35 \mathrm{mmHg}$ overnight. Then it was placed in the MAGNUM II reactor from ERTEC Company (PTFE vessel of MHR Ertec, figure 1).

The power of microwave reactor was adjusted to $100 \mathrm{~W}$. The processes were performed at a temperature of $50^{\circ} \mathrm{C}$, maximum pressure allocated changed to $2 \mathrm{~atm}$. The time

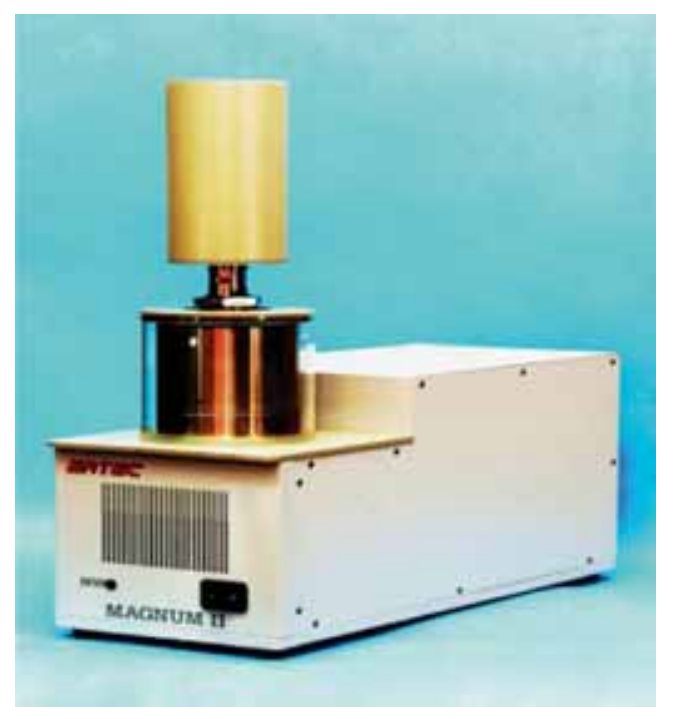

Figure 1. Microwave hydrothermal reactor. adjusted was 4 min for sample 1 and 25 min for sample 2 in the reactor. It was repeated at a temperature of $180^{\circ} \mathrm{C}$ for 10 and $60 \mathrm{~min}$ to fabricate another two samples which were named as samples 3 and 4, respectively. High-resolution transmission electron microscopy (HR-TEM, FEI Tecnai F30) was employed to study the morphology of graphene sheets. Optical absorption spectra were recorded using a Perkin Elmer UV-vis spectrometer. Fourier transform infrared spectra (FTIR) were acquired on a Nicolet 6700 FTIR spectrometer. Infrared spectra were recorded on a FTIR spectrometer (100 N model), equipped with deuterated triglycine sulphate (DTGS) detector, and $\mathrm{KBr}$ beam splitter. X-ray photoelectron spectroscopy (XPS) was used to study the chemical state variations of the GO sheets on application of the microwave. The data were obtained through a hemispherical analyzer supplied by an $\mathrm{Al} \mathrm{K} \alpha \mathrm{X}$-ray source $(h v=1486.6 \mathrm{eV})$ operating at a vacuum better than $10^{-7} \mathrm{~Pa}$. For quantitative analyses, the XPS peaks were de-convoluted by using Gaussian components after a Shirley background subtraction. Quantitative elemental compositions were obtained based on peak area ratio of the XPS core levels modified by the sensitivity factor (SF) of each element in XPS. Thermogravimetric analysis (TGA) was performed simultaneously using a SDT Q600 under $\mathrm{N}_{2}$ flow rate of $40 \mathrm{ml} \mathrm{min}^{-1}$ and heating rate of $15^{\circ} \mathrm{C} \mathrm{min}^{-1}$. Raman spectra were acquired using a Dispersive Raman Microscope Senterra-2009 at excitation wavelength of $785 \mathrm{~nm}$.

\section{Results and discussion}

Morphological and structural features of the synthesized GO powders were studied by HR-TEM. Figure 2 exhibits sheet structures of multi-layer GO sheets. The high transparency of the sample indicates few layered structure and well dispersion of the GO layers. Using figure $2 b$ the thickness of the multi-layer graphene is $4 \mathrm{~nm}$ then each sheet is $\sim 0.8 \mathrm{~nm}$ which is consistent with the typical thickness of singlelayer GO, and the distance between each two stacked layers is $\sim 2 \mathrm{~nm}^{25}$

The UV-visible spectra of the GO suspension before and after the microwave for different temperatures and exposure times are shown in figure 3. The position of the absorption peak of the spectra indicates the degree of deoxygenation. The GO suspension displays an absorption peak at $\sim 230 \mathrm{~nm}$ due to the $\pi-\pi^{*}$ of aromatic $\mathrm{C}=\mathrm{C}$ transition in the aromatic
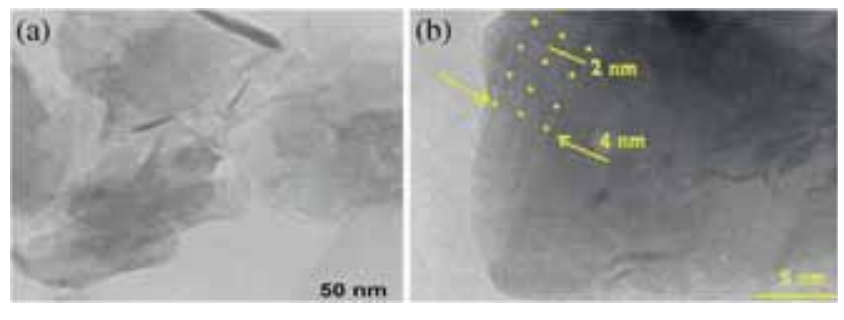

Figure 2. HR-TEM images of (a) multi-layer GO sheets and (b) the maximum thickness of stacked GO sheets. 


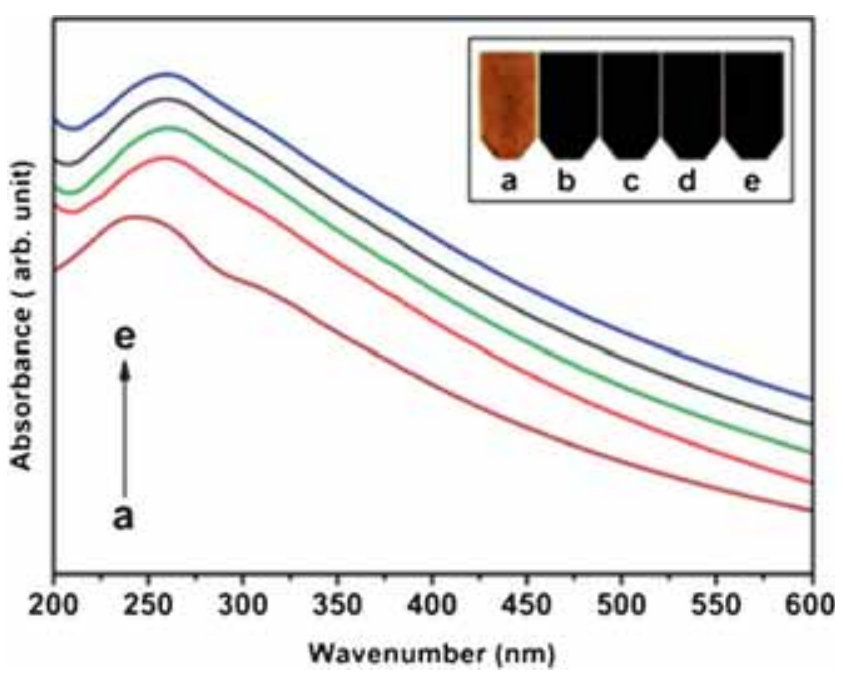

Figure 3. Optical absorption spectra of (a) GO suspension (0.02 $\mathrm{mg} 1^{-1}$ in water) and reduced GO, (b) sample 1 with 4 min, (c) sample 2 with $25 \mathrm{~min}$, (d) sample 3 with $10 \mathrm{~min}$ and (e) sample 4 with $60 \mathrm{~min}$ in microwave hydrothermal reactor.

bands. ${ }^{26-28}$ The more the $\pi-\pi^{*}$ transitions (conjugation), the less the energy needed to be used for the electronic transition. After applying MHR the absorption peak at $230 \mathrm{~nm}$ is red-shifted to $270 \mathrm{~nm}$, indicating that the electronic conjugation inside the reduced graphene sheets was restored after reduction of graphene. The reduction of GO to graphene is necessary to recover the conjugated network and electrical conductivity. The red shift together with the increase of the absorption in the whole spectral region after MHR indicates deoxygenation and removal of the carbocyclic and carbonyl groups from surface of the GO sheets. ${ }^{29,30}$ Upon reduction $\pi$-electron concentration increased reflecting structural ordering, which is consistent with the restoration of $\mathrm{sp}^{2}$ carbon and possible rearrangement of atoms. Furthermore, a shoulder at $\sim 300 \mathrm{~nm}$ is observable which can be attributed to $\mathrm{n} \rightarrow \pi^{*}$ transitions of the carbonyl groups $(\mathrm{C}=\mathrm{O})$. By using the microwave, the optical absorption of the samples 1-4 is gradually increased; indicating progressive restoration of the electronic conjugation within the RGO sheets. ${ }^{29}$ This is also in consistent with the colour change of the suspension from light brown for the GO to black for the microwave-RGO which is depicted in figure 3 .

Reduction of the oxygen-containing groups of the GO treated by the microwave was studied by using FTIR spectroscopy (figure 4). Absorption bands for the GO sheets was found to be located at $3340 \mathrm{~cm}^{-1}(\mathrm{O}-\mathrm{H}$ stretching band), $1730 \mathrm{~cm}^{-1}(\mathrm{C}=\mathrm{O}$ stretching band $), 1630 \mathrm{~cm}^{-1}$ (skeletal vibrations of aromatic domains), $1401 \mathrm{~cm}^{-1}$ (bending absorption of carboxyl group $\mathrm{O}=\mathrm{C}-\mathrm{O}$ ), $1226 \mathrm{~cm}^{-1}$ (O-H bending vibrations) and $1045 \mathrm{~cm}^{-1}(\mathrm{C}-\mathrm{O}$ stretching vibrations), consistent with the previous reports. ${ }^{31}$

Figure 4 shows that in all samples especially sample 4 the main absorption peaks of oxygen-containing functional groups (including $\mathrm{O}-\mathrm{H}, \mathrm{C}=\mathrm{O}$ and $\mathrm{C}-\mathrm{O}$ bonds) are

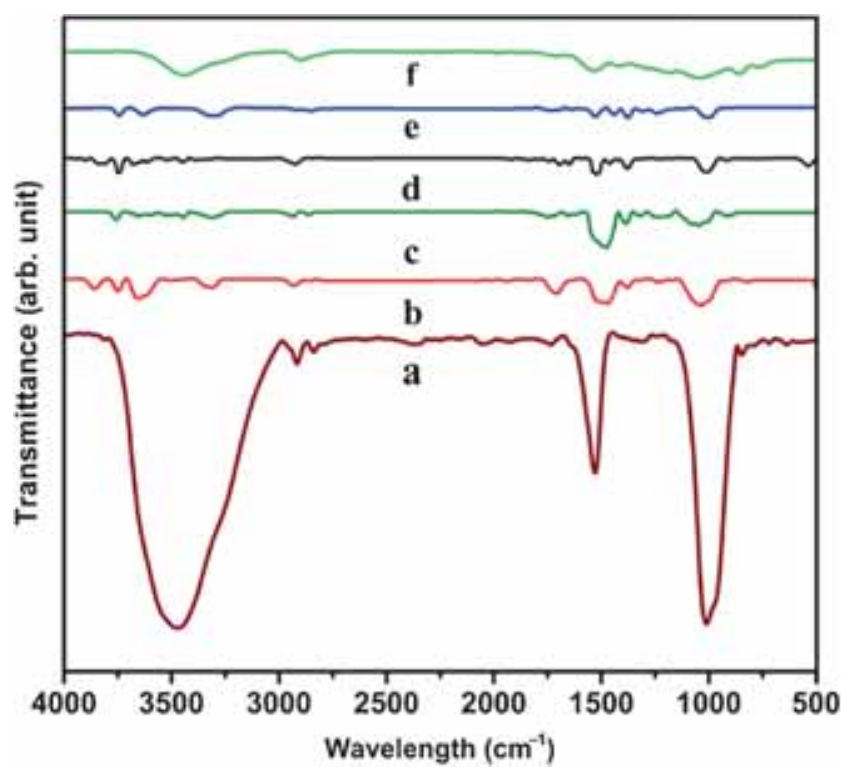

Figure 4. FTIR spectra of (a) GO and reduced GO, (b) sample 1 with $4 \mathrm{~min}$, (c) sample 2 with $25 \mathrm{~min}$, (d) sample 3 with $10 \mathrm{~min}$, (e) sample 4 with $60 \mathrm{~min}$ in microwave hydrothermal reactor and (f) using hydrazine.

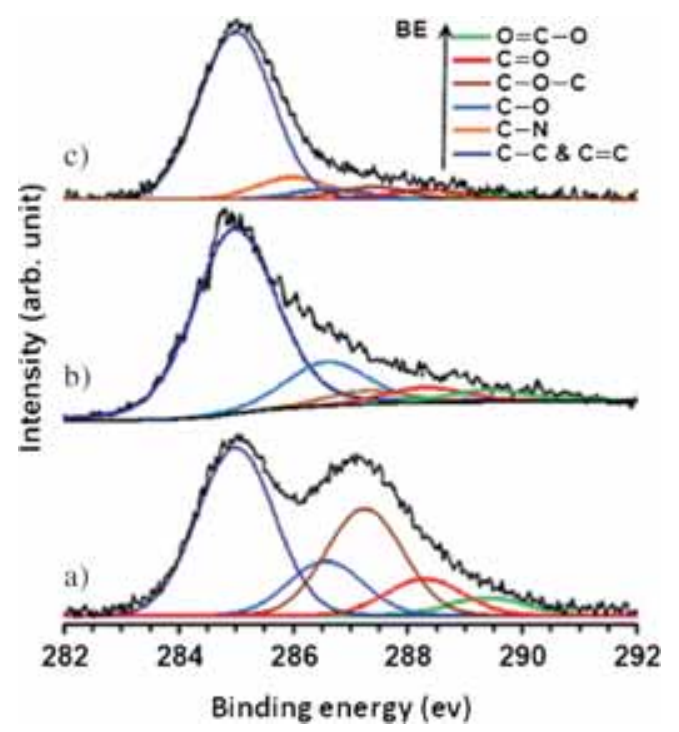

Figure 5. XPS peak deconvolution of $\mathrm{C}(1 \mathrm{~s})$ core level of (a) GO, (b) microwave-RGO and (c) hydrazine-RGO.

entirely vanished, representing great effective deoxygenating of GO sheets using MHR. On the other hand, reduction by hydrazine displays decrease in oxygen groups at 990 1100 and $3140 \mathrm{~cm}^{-1}$. Clearly hydrazine has a great influence such as MHR but it is hazardous. Other reports have shown the reduction of GO by microwave, ${ }^{32}$ but in our method different samples were reduced completely without showing significant oxygen groups from 4000 to $500 \mathrm{~cm}^{-1}$.

Figure 5 shows XPS peak deconvolution of $\mathrm{C}(1 \mathrm{~s})$ core level of the microwave-RGO sheets, as compared with the peaks of the GO ones. In the peak deconvolution, 
the peak centred at $285.0 \mathrm{eV}$ was assigned to the $\mathrm{C}-\mathrm{C}$ and $\mathrm{C}=\mathrm{C}$ bonds. The other deconvoluted peaks located at the binding energies of 286.6, 287.3, 288.3 and $289.4 \mathrm{eV}$ are attributed to the $\mathrm{C}-\mathrm{OH}, \mathrm{C}-\mathrm{O}-\mathrm{C}, \mathrm{C}=\mathrm{O}$ and $\mathrm{O}=\mathrm{C}-$ $\mathrm{OH}$ oxygen-containing functional groups, respectively. After reduction the GO sheets by microwave, the peaks relating to the oxygen-containing functional groups significantly reduced. Comparison between figure $5 \mathrm{~b}$ and $\mathrm{c}$ indicates that deoxygenation efficiency of the GO sheets by microwave was as good as with the efficiency obtained by hydrazine (as a strong reducer) at the same $\mathrm{pH}$ of the solution $(\sim 9)$. However, figure $5 \mathrm{c}$ presents another peak component at $286.0 \mathrm{eV}$ which can be attributed to the formation of $\mathrm{C}-\mathrm{N}$ bond on surface of the hydrazine-RGO sheets. ${ }^{33}$ In contrast, no peak relating to the formation of $\mathrm{C}-\mathrm{N}$ bond was found in the XPS of the microwave-RGO samples. Based on the SF-modified peak area ratios of the XPS $\mathrm{C}(1 \mathrm{~s})$ and $\mathrm{O}(1 \mathrm{~s})$ core levels, the $\mathrm{O} / \mathrm{C}$ ratios of the as-prepared GO sheets were calculated as $49 \%$, whereas for the microwave-RGO and hydrazine-RGO sheets it decreased to 17 and $15 \%$, respectively. These results confirmed that the deoxygenation efficiency of the microwave reduction method was comparable with the standard efficiency expected by hydrazine. It can be compared with microwave thermal reduction of GO suspension in DMAc/ $\mathrm{H}_{2} \mathrm{O}$, which has reported efficiency of $18.3 \% .^{34}$

TGA was used to illustrate the removal of oxygen groups. Figure 6 shows TGA heating curves of GO, RGO reduced by hydrazine, and RGO after microwave. All the samples were analysed under $\mathrm{N}_{2}$ atmosphere with a heating rate of $15^{\circ} \mathrm{C} \mathrm{min}^{-1}$. GO, hydrazine-RGO and microwave-RGO displays $5 \%$ and $3 \%$ weight loss around $100^{\circ} \mathrm{C}$ due to water removal, respectively. For GO and hydrazine-RGO sheets, a sudden weight loss (totally $\sim 37-18 \%$ ) was observed between 180 and $300^{\circ} \mathrm{C}$, corresponding to the removal of more stable oxygen-containing functional groups from surface of the sheets, although removal of the oxygen bonds are completed in the temperature range of $180-300^{\circ} \mathrm{C}$. Also, for microwave-RGO a small abrupt weight loss (only 4\%) was considered around $180-300^{\circ} \mathrm{C}$, related to remaining oxygen functional groups on GO sheets. The TGA curve clearly demonstrates the MHR influences in reduction procedure which approximately remove the oxygen functional groups on the sheets and it is impressively better than hydrazine.

Other previous studies have reported reduction by the microwave treatment was shown infinitesimal mass loss at around $180-300^{\circ} \mathrm{C}$ and most of the mass loss occurs at about $500^{\circ} \mathrm{C}$ which indicates the nearly completion of the reduction process of $\mathrm{GO} .{ }^{35,36}$

Raman spectra of GO display two broad peaks at 1303 and $1590 \mathrm{~cm}^{-1}$, corresponding to the D and the $\mathrm{G}$ bands, respectively (figure 7). After reduction a slight shift in both the D and the $\mathrm{G}$ bands are observed and these two peaks transmitted to upper wavenumber which are located at 1309 and 1599 $\mathrm{cm}^{-1}$ for the $\mathrm{D}$ and the $\mathrm{G}$ peaks, respectively. This result has been attributed to the stress which builds up during the cool-down process after applying MHR. ${ }^{37}$ In general, tensile

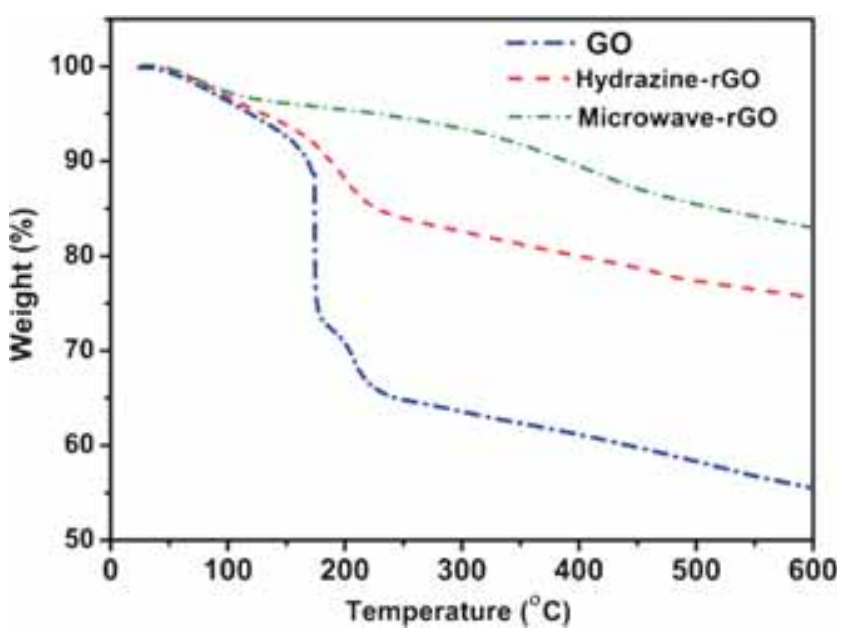

Figure 6. TGA plot of GO, hydrazine-RGO and RGO after microwave.

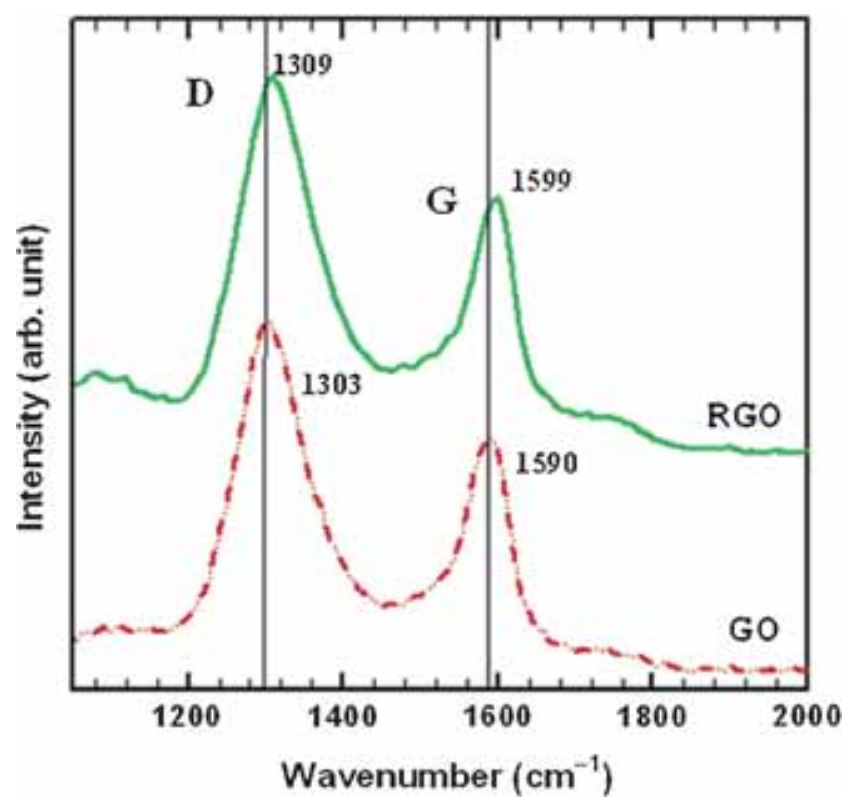

Figure 7. Raman spectra for GO and RGO after microwave.

strain induces phonon softening (red shift) and compression causes phonon hardening (blue shift). ${ }^{38}$

The $I_{\mathrm{D}} / I_{\mathrm{G}}$ ratio in Raman spectroscopy can be used to evaluate the distance between defects in graphene which is about 1 for pristine graphene and increases with the decrease in average size of the $\mathrm{sp}^{2}$ domain and average crystalline size $\left(L_{\mathrm{a}}\right): 39$

$$
L_{\mathrm{a}}=\left(2.4 \times 10^{-10}\right) \lambda_{\text {laser }}^{4}\left[\frac{I(D)}{I(G)}\right]^{-1},
$$

where $L_{\mathrm{a}}$ is the average crystalline size and $\lambda$ the laser wavelength in $\mathrm{nm}$. The calculated average crystalline sizes of GO and RGO are 22.6 and $22.08 \mathrm{~nm}$, respectively, indicating the decrease in average crystalline size of RGO compared with GO. 
After applying MHR the Raman spectrum displayed an increase in the intensity of the $\mathrm{D}$ band compared with that of the $\mathrm{G}$ band, but the ratio of $I_{\mathrm{D}} / I_{\mathrm{G}}$ are 1.5 and 1.46 for $\mathrm{GO}$ and RGO, respectively (figure 7 ). The $I_{\mathrm{D}}$ peak intensity is a measurement of disorder degree defects which arise from vacancies, distortion and edges. ${ }^{40}$ In order to find the origin of increase in the D peak, density of defect can be calculated by relation ${ }^{41}$

$$
n_{\mathrm{D}}^{2}\left(\mathrm{~cm}^{-1}\right)=\frac{5.9 \times 10^{14}}{E_{\mathrm{L}}^{4}}\left[\frac{I(D)}{I(G)}\right]^{-1} .
$$

Using this formula, the same $n_{\mathrm{D}}$ for GO and RGO was obtained, which means the increase in the $D$ intensity is not due to the increase in defects. It would be because of the decrease in the size of sheets and its edges after reduction. Also there is no strong peak around $1620 \mathrm{~cm}^{-1}$, the so-called $D^{\prime}$ peak, which is attributed to defected graphite. ${ }^{42}$

\section{Conclusions}

GO sheets were synthesized by Hummers' method. MHR was applied to reduce graphene oxide. Different temperatures $50^{\circ} \mathrm{C}$ and $180^{\circ} \mathrm{C}$ were applied to fabricate four samples with 4, 10, 25 and 60 min exposure times. The effective reduction of the GO sheets was confirmed by UV-visible, FTIR, XPS and TGA. Using XPS analysis, the O/C ratio of the GO sheets reduced from 49 to $17 \%$ after microwave. Because, hydrazine reduced the $\mathrm{O} / \mathrm{C}$ ratio down to $15 \%$, MHR reduction method can be considered as a serious potential competitor for hydrazine.

Raman spectroscopy results demonstrated that average crystalline sizes were 22.6 and $22.08 \mathrm{~nm}$ and the ratio of $I_{\mathrm{D}} / I_{\mathrm{G}}$ were 1.5 and 1.46 for GO and RGO, respectively. Density of defect was calculated and the result showed that the increase in the $\mathrm{D}$ peak intensity was not due to the increase in defects. It would be because of decrease in the size of sheets after reduction. Furthermore, there was no peak around $1620 \mathrm{~cm}^{-1}$, the so-called $D^{\prime}$ peak, which was attributed to defected GO. Therefore MHR can be used to reduce GO as an environment-friendly reduction.

\section{Acknowledgement}

Salimeh Kimiagar would like to thank Islamic Azad University, Central Tehran Branch, for financial support of this research.

\section{References}

1. Zhu J, Xu M, Meng X, Shang K, Fan H and Ai S 2012 Process. Biochem. 472480

2. Ghadim E E, Manouchehri F, Soleimani G, Hosseini H, Kimiagar S and Nafisi S 2013 PLoS ONE 879254

3. Kaloni $\mathrm{T}$ P, Cheng $\mathrm{Y}$ C, Upadhyay Kahaly $\mathrm{M}$ and Schwingenschlögl U 2012 Chem. Phys. Lett. 53429
4. Du W, Zhou B and Jiang X 2014 Chem. Phys. Lett. 595-596 1

5. Kou R, Shao Y, Mei D, Nie Z, Wang D, Wang C, Viswanathan W, Park S, Aksay I A, Lin Y, Wang Y and Liu J $2011 \mathrm{~J}$. Am. Chem. Soc. 1332541

6. Li Y, Li X, Dong C, Qi J and Han X 2010 Carbon 483427

7. Sun Z, James D K and Tour J M 2011 J. Phys. Chem. Lett. 2 2425

8. Marcano D C, Kosynkin D V, Berlin J M, Sinitskii A, Sun Z, Slesarev A, Alemany L B, Lu W and Tour J M 2010 Nano 4 4806

9. Tang X-Z, Li W, Yu Z-Z, Rafiee M A, Rafiee J, Yavari F et al 2011 Carbon 491258

10. Tung V C, Allen M J, Yang Y and Kaner R B 2009 Nat. Nanotechnol. 425

11. Park S, An J H, Jung I W, Piner R D, An S J, Li X S et al 2009 Nano Lett. 91593

12. Wang G X, Yang J, Park J, Gou X L, Wang B, Liu H et al 2008 J. Phys. Chem. C 1128192

13. Si Y and Samulski E T 2008 Nano Lett. 81679

14. Amal Raj M and John A S 2013 J. Phys. Chem. C 117 4326

15. Becerril H A, Mao J, Liu Z, Stoltenberg R M, Bao Z and Chen Y 2008 ACS Nano 2463

16. Wang Q, Chen X, Yu K, Zhang Y and Cong Y 2013 J. Hazard. Mater. 246-247 135

17. Trusovas R, Ratautas K, Račiukaitis G, Barkauskas J, Stankevičienè I, Niaura G et al 2013 Carbon $\mathbf{5 2} 574$

18. Wang L, Li X, Teng W, Zhao Q, Shi Y, Yue R et al 2013 J. Hazard. Mater. 244-245 681

19. Zhu Y, Murali S, Stoller M D, Velamakanni A, Piner R D and Ruoff R S 2010 Carbon $\mathbf{4 8} 2118$

20. Yan Y, Hojamberdiev M, Xu Y, Wang J and Luan Z 2013 Mater. Chem. Phys. 139298

21. Wolska E, Sibera D, Witkowski B S, Yatsunenko S A and Pełech I 2011 Acta Phys. Polonica A 120908

22. Ma E, Li J, Zhao N, Liu E, He C and Shi C 2013 Mater. Lett. 91209

23. Zheng J, Liu H T, Wu B, Di C A, Guo Y L, Wu T, Yu G, Liu Y Q and Zhu D B 2012 Sci. Rep. 2662

24. Wei T, Fan Z J, Luo G L, Zheng C and Xie D S 2009 Carbon 47337

25. Ghadim E E, Rashidi N, Kimiagar S, Akhavan O, Manouchehri F and Ghaderi E 2014 Appl. Surf. Sci. 301183

26. Ang P K et al 2009 ACS Nano 33587

27. Luo Z T, Lu Y, Somers L A and Johnson A T C 2009 J. Am. Chem. Soc. 131898

28. Mei Q, Zhang K, Guan G, Liu B, Wang S and Zhang Z 2010 Chem. Commun. 467319

29. Li D, Muller M B, Gilje S, Kaner R B and Wallace G G 2008 Nat. Nanotechnol. 3101

30. Chang H-W, Tsai Y-C, Cheng C-W, Lin C-Y and Wu P-H 2012 Electrochem. Commun. 2337

31. Stankovich S, Piner R D, Nguyen S T and Ruoff R S 2006 Carbon 443342

32. Pokharel P, Truong Q-T and Lee D S, 2014 Composites Part B: Engineering 64187

Slesarev L B A, Wei Lu and Tour J M 2010 Nano 44806 
33. Esfandiar A, Akhavan O and Irajizad 2011 J. Mater. Chem. 21 10907

34. Chen W, Yan L and Bangal P R 2010 Carbon 481146

35. Dang T T, Pham V H, Hur S H, Kim E J, Kong B S and Chung J S 2012 J. Colloid Interface Sci. 37691

36. Han Y, Luo Z, Yuwen L, Tian J, Zhu X and Wang L 2013 Appl. Surf. Sci. 266188

37. Lee D S, Riedl C, Krauÿ B, von Klitzing K, Starke U and Smet J H 2008 Nano Lett. 84320
38. Frank O, Tsoukleri G, Parthenios J, Papagelis K, Riaz I, Jalil R, Novoselov K S and Galiotis C 2010 ACS Nano 43131

39. Pimenta M A, Dresselhaus G, Dresselhaus M S, Cançado L G, Jorio A and Saito R 2007 Phys. Chem. Chem. Phys. 91276

40. Tuinstra F and Koenig J L 1970 J. Chem. Phys. 531126

41. Ferrari A C and Robertson J 2000 Phys. Rev. B 6114095

42. Ferrari A C, Meyer J C, Scardaci V, Casiraghi C, Lazzeri M, Mauri F, Piscanec S, Jiang D, Novoselov K S, Roth S and Geim A K 2006 Phys. Rev. Lett. 97187401 